\title{
The Optimal Robust Portfolio Model Based on CDaR
}

\author{
Xing $\mathrm{Yu}$ \\ Department of Mathematics \& Applied Mathematics of Hunan University of humanities \\ Science and technology \\ Loudi,China \\ E-mail: hnyuxing@163.com
}

\begin{abstract}
This paper proposed a robust portfolio model, using CDaR to measure portfolio risk. Due to uncertainty parameter, we constructed ellipsoidal uncertainty set as the parameters uncertainty set to maximize the portfolio return. We verified the operable of the model with numerical simulation. The result shows that the risk is higher compared to without robust case, which is helpful to cautious investment for investors.
\end{abstract}

\section{Keywords- portfolio; CDaR; robust; CVaR; Monte Carlo}

\section{Introduction}

The bank for International Settlements in 1993 put forward the " value at risk" [1] ( Value-at-Risk, VaR for brief) concept. It is said that the risk value after a time interval having a maximum possible loss of confidence, VaR method can not only calculate risk at advance, but also can measure all of overall portfolio risk, relatively speaking, VaR is relatively simple, easy to understand, so more and more financial institutions using $\mathrm{VaR}$ to measure market risk, VaR gradually became metric financial risk as the mainstream methods, many financial institutions and business departments in the investment choices, are considered to meet the VaR constraint.

Although VaR is very popular, but in mathematics, it has certain limitations, such as not having time added new, nonconvex and multipolar value. These properties are affected by VaR as a target for the portfolio selection. In view of the $\mathrm{VaR}$ of these defects, the theorists proposed conditional value at risk ( $\mathrm{CVaR}$ ), it is more than VaR of the additional loss of the average value of [2]. CVaR is a coherent risk measure, it can be solved by linear programming to optimize. A large body of literature ( such as the Rockafellar and Uryasev, 2000; Rockafellar and Uryasev, 2002; Pflug, 2000; Gaivoronski and Pflug, 2001) minimize adhesion risk measurement $\mathrm{CVaR}$ to establish the optimal portfolio model [3-6].

In solving the minimization of CVaR problem, due to the calculation is relatively complex, the [7] defines a discrete case of simple form, the portfolio represented in a discrete form. CVaR model is a coherent risk measure model, with subadditive, but when the portfolio loss density function is not continuous functions, $\mathrm{CVaR}$ model is no longer a coherent risk measure model, that is, CVaR model is not the generalized coherent risk measure model, the need for certain improvements [8]. Based on the CDaR portfolio model can refer to [9]. however, they are all in determining case discusses the optimal combination problem, that is using historical data to said future features, since the future is unknown and uncertain data, and make a portfolio for future decision-making, model depends largely on the result of uncertainty the parameters, such as the stock return and volatility, a large number of facts [10] model parameters of small changes can result in model results to be quite different, even severe consequences, therefore, in portfolio problem it is necessary to fully consider the uncertainty of parameters. To solve the problem of parameter uncertainty in two ways, one is stochastic programming, this method in the calculation of the more complicated, and the robust optimization method by many scholars of the pro-Lai [11-13].Anna

Grazia Quaranta[14] proposed based on minimizing the combination CVaR robust optimal portfolio model, improved Rockafellar and Uryasev 's model based on stochastic programming optimal portfolio model. But the model in order to facilitate the combination of income is taken as the discrete case, it destroys the CVaR consistency. This paper uses CDaR describe the portfolio risk, considers the parameter uncertainties, robust optimal portfolio model is established, and through empirical study it is confirmed the feasibility of the model, and the results with CVaR case comparison shows the validity of the model.

\section{THE DEFINITION OF CDAR}

Suppose $w(x, t)$ is the return of portfolio at time $\mathrm{t}$, the investment shares are $x=\left(x_{1}, x_{2} \cdots x_{n}\right)$, the drop function is defined at time $\mathrm{t}$ as[15] $D(x, t)=\max _{0 \leq \tau \leq t}\{w(x, \tau)\}-w(x, t)$, the corresponding

\section{$\mathrm{CDaR}$ is defined as}

$\Delta_{\alpha}(x)=\min _{\xi}\left\{\xi+\frac{1}{(1-\alpha) T} \int_{0}^{T}[D(x, t)-\xi]^{+} d t\right\}(1)$

Due to the distribution of portfolio return is generally unknown, it is difficult to calculate $\Delta_{\alpha}(x)$ using formula

(1) , so it is necessary to transfer the problem to discrete case, and the CDaR function is: 
$\Delta_{\alpha}(x)=\min _{\xi}\left\{\xi+\frac{1}{(1-\alpha) J} \sum_{k=1}^{J}\left(\max _{1 \leq j \leq k} y_{j} x-y_{k} x-\xi\right)^{+}\right\}$

Let $w_{k}=\max _{1 \leq j \leq k} y_{j}-y_{k}$, and

$$
\Delta_{\alpha}(x)=\min _{\xi}\left\{\xi+\frac{1}{(1-\alpha) J} \sum_{k=1}^{J}\left(w_{k} x-\xi\right)^{+}\right\}(3)
$$

\section{THE OPTIMAL PORTFOLIO MODEL BASED ON CDAR}

For investors, their goal is to meet the expected return under the premise to minimize the portfolio risk, suppose that there are $\mathrm{N}$ assets, the expected rate of return of investors is $\mathrm{C}$, and limit shorting sell, then set up portfolio model (P1) as following:

$$
\min _{\xi, x}\left\{\xi+\frac{1}{(1-\alpha) J} \sum_{k=1}^{J}\left(w_{k} x-\xi\right)^{+}\right\}
$$

$$
\text { s.t }\left\{\begin{array}{l}
\sum_{i=1}^{N} r_{i} x_{i} \geq C \\
x_{1}+x_{2}=1 \\
x_{1} \geq 0 \\
x_{2} \geq 0
\end{array}\right.
$$

\section{The SOlution TO THE MODEL BASED ON CDAR}

In order to calculate conveniently, we introduce auxiliary variable $z_{k}$, and transfer the model to linear model (P2) :

$$
\min _{\xi, x}\left\{\xi+\frac{1}{(1-\alpha) J} \sum_{k=1}^{J} z_{k}\right\}
$$

$$
\text { s.t }\left\{\begin{array}{c}
z_{k} \geq w_{i k} x-\xi \\
z_{k} \geq 0, k=1,2 \cdots J \\
\sum_{i=1}^{N} r_{i} x_{i} \geq C \\
x_{1}+x_{2}=1 \\
x_{1} \geq 0 \\
x_{2} \geq 0
\end{array}\right.
$$

\section{A. The uncertain sets}

Because of the expected rate of return are unable to accurately predict, if the use of the historical return to represent the future returns it will lead to larger deviations, and the expected rate of return of a small changes may lead to asset portfolio allocation of great changes, so we need to introduce parameter uncertainty set, the model is transformed for the robust optimization problem.

Construct ellipsoidal uncertainty set[16]:

$$
\Theta_{r}=\left\{r:(r-\mu)^{T} \Sigma^{-1}(r-\mu) \leq \delta^{2}\right\}
$$

where $\delta=\sqrt{\frac{p}{1-p}}, p \in[0,1)$

parameter $p$ describe the risk aversion coefficient of investors, $\mu$ is sample mean, $\Sigma$ is sample variance.

B.The robust optimization model (P3)

$$
\min _{\xi, x}\left\{\xi+\frac{1}{(1-\alpha) J} \sum_{k=1}^{J} z_{k}\right\}
$$

$$
\text { s.t }\left\{\begin{array}{c}
\quad z_{k} \geq w_{i k} x-\xi \\
\quad z_{k} \geq 0, k=1,2 \cdots J \\
\min _{r} \sum_{i=1}^{N} r_{i} x_{i} \geq C \\
(r-\mu)^{T} \Sigma^{-1}(r-\mu) \leq \delta^{2} \\
x_{1}+x_{2}=1 \\
x_{1} \geq 0 \\
x_{2} \geq 0
\end{array}\right.
$$

where $w_{i k}=\max _{1 \leq j \leq k} y_{i j}-y_{i k}$, let $\alpha=0.9$, so $\delta=2$. $p=0.8$

In our model, there are three uncertain inputs should be considered variance $\sigma$ and mean $\mu$, in some literatures, it is assumed that the estimate covariance is accurate such that there is no uncertainty about it. According to[17], which show that the error in mean estimation by far out weights the estimation error in covariance. That is, although $\sigma$ is uncertain, in this paper, we suppose it is certain, which is estimated by sample variance $S$.

Secondly, we consider the uncertain set for return mean. We define $r^{\prime}$ is the estimation of real value $r$, the uncertainly set $I$ as $I=\left\{r: r_{i}^{\prime}-s_{i} \leq r_{i} \leq r_{i}^{\prime}+s_{i}\right\}$ for mean. According to Anna[23], the robust counterpart: 


$$
\min \sum r_{i} x_{i} \geq r_{p}
$$

can be transferred to the following form:

$$
\left\{\begin{array}{c}
\sum r_{i}^{\prime} x_{i}-\sum s_{i} m_{i} \geq r_{p} \\
m_{i} \geq x_{i} \\
s_{i} \geq 0
\end{array}\right.
$$

So, the final model is

$(P 4)$

$$
\min _{\xi, x}\left\{\xi+\frac{1}{(1-\alpha) J} \sum_{k=1}^{J} z_{k}\right\}
$$

$$
\text { s.t }\left\{\begin{array}{c}
z_{k} \geq w_{i k} x-\xi \\
z_{k} \geq 0, k=1,2 \cdots J \\
\sum r_{i}^{\prime} x_{i}-\sum s_{i} m_{i} \geq C \\
m_{i} \geq x_{i} \\
s_{i} \geq 0 \\
(r-\mu)^{T} \Sigma^{-1}(r-\mu) \leq \delta^{2} \\
x_{1}+x_{2}=1 \\
x_{1} \geq 0 \\
x_{2} \geq 0
\end{array}\right.
$$

Obviously, the model is a problem of large scale linear programming problem, although, it can be solved using LINGO.

\section{NUMERICAL STUDY}

For the convenience of research, we only a select two stocks in portfolio as the research object, the two shares information as follows: $S_{1}(0)=15.59, S_{2}(0)=13.71$, $\mu_{1}=11 \%, \sigma_{1}=26.86 \% ; \mu_{2}=8.05 \%, \sigma_{2}=16.3 \%$

It simulated the two stocks price through Monte Carlo . if investment time for one years, that is 240 trading days, solving model ( P3 ) to get the objective function value is 15.6. while no robust case we derive the solution of the model is 13.2, compared to the robust case it leads lower risk, this is due to the consideration of parameter changes effecting on the solutions of the model, it is good for investors cautious investment .

As a new risk measurement tool, $\mathrm{CDaR}$ model has more advantages in insurance fund investment risk management than the $\mathrm{VaR}$ and $\mathrm{CVaR}$ model .First, the portfolio optimization model, the solving process does not assume the yields of specific distribution, thus it is widely applicable; secondly, optimization model by appropriately incorporating auxiliary variables into simple linear programming problem, solving convenient. Considering the influence of model parameters on the portfolio; this paper also introduce robust optimization to the model, a simulation example is robust case depicts the risk to be higher than no robust, which help improving investors cautious investment. In the model according to the investor's risk preference, to adjust the parameters, so as to achieve the purpose of different investors, thus suitable for different risk preferences of investors to make investment decisions and management.

\section{ACKNOWLEDGMENT}

This research is supported by Department of Education Topics of Hunan Province. (NO 12C0749)

\section{REFERENCES}

[1] Weilun $\mathrm{Xu}$, To discuss that under Mean-Variance model, the influence of $\mathrm{VaR}$ or $\mathrm{CVaR}$ for portfolio selection[D] , Tsinghua University,1994.

[2] Yongchun Xu,Yuelin Gao. CVaR for finance risk management and empire analysis. Search.,vol 2.2009,pp.11-13.

[3] Rockafellar, R.T., Uryasev, S., Optimization of conditional value at risk. J. Risk 3 (February). 2000.pp. 21-41.

[4] Rockafellar, R.T., Uryasev, S., Conditional value at risk for general loss distributions. J. Bank. Finance. (26/7), 2002.pp.1443-1471.

[5] Pflug, G.Ch., 2000. Some remarks on the value at risk and the conditional value at risk. In: Uryasev, S. (Ed.), Probabilistic Constrained Optimization:Methodology and Applications. Kluwer Academic Publishers

[6] Gaivoronski, A.A., Pflug, G., Value at risk in portfolio optimization: properties and computational approach.vol 16, 2001..pp.241-252.

[7] Uryasev Stanislav. Conditional Value - at - Risk, Optimization Algorithms and Applications . Financial Engineering News ,vol14,2000.pp.423-441.

[8] Zhongwu,Yuxi Wang . Based on the CDaR theory of social security fund investment and risk management model construction .Statistics and decision-making. vol 2.2009.pp.45-47.

[9] A.Chekhlov,S.Uryasev and M. Zaharankin, Drawdown. Measure in portfolio optimization. International Journal of Theoretical and Applied Finance,vol 1,2005.pp.34-37.

[10] Bertsimas D, Brown D.Caramanis C. Theory and applicationss of robust optimization, Working paper, 2007.

[11] BenTal, A., Nemirovski, A., Robust convex optimization. Math. Oper. Res. 23 (4). 1998.pp.769-805.

[12] BenTal, A., Nemirovski, A., Robust optimization - methodology and applications. Math. Program., Ser. B 92.2002.pp.453-480.

[13] Goldfarb, D., Iyengar, G., Robust portfolio selection problems. Math. Oper. Res.. vol 6,2001.pp.367-385.

[14] Anna Grazia Quaranta *, Alberto Zaffaroni. Robust optimization of conditional value at risk and portfolio selection. Journal of Banking \& Finance 32,2008. pp.2046-2056.

[15] Zhongwu. Based on the CDaR theory of social security fund investment and risk management model .Commercial research. vol 7,2009.pp.106-108.

[16] BenTal, A., Nemirovski, A., Robust optimization - methodology and applications. Math. Program., Ser. B 92, 2002.pp.453-480.

[17] Anna G.Q., Alberto z. Robust optimization of conditional value at risk and portfolio selection. Journal of Banking \& Finance vol 32,pp.2046-2056,2008. 